\title{
Friedel Oscillations in Holographic Metals
}

\author{
V. Giangreco M. Puletti, ${ }^{a}$ S. Nowling, ${ }^{b}$ L. Thorlacius, ${ }^{b, c}$ and T. Zingg ${ }^{b, c}$ \\ ${ }^{a}$ Department of Fundamental Physics, \\ Chalmers University of Technology, 41296 Göteborg, Sweden \\ ${ }^{b}$ Nordita \\ Roslagstullsbacken 23, SE-106 91 Stockholm, Sweden \\ ${ }^{c}$ University of Iceland, Science Institute \\ Dunhaga 3, IS-107 Reykjavik, Iceland \\ E-mail: marotta@chalmers.se, nowling@nordita.org, \\ larus@nordita.org, zingg@nordita.org
}

\begin{abstract}
In this article we study the conditions under which holographic metallic states display Friedel oscillations. We focus on systems where the bulk charge density is not hidden behind a black hole horizon. Understanding holographic Friedel oscillations gives a clean way to characterize the boundary system, complementary to probe fermion calculations. We find that fermions in a "hard wall" AdS geometry unambiguously display Friedel oscillations. However, similar oscillations are washed out for electron stars, suggesting a smeared continuum of Fermi surfaces.
\end{abstract}

KEYwords: AdS-CFT correspondence, Friedel Oscillations 


\section{Contents}

1 Introduction 1

2 Friedel Oscillations $\quad 4$

2.1 Perturbative Friedel Oscillations in $2+1$ Dimensions 5

3 Friedel Oscillations in an AdS Hard Wall Geometry 6

3.1 Preliminaries 6

$\begin{array}{lll}3.2 & \text { Mean Field Susceptibility } & 7\end{array}$

3.3 Polarization Corrections 8

3.3.1 Illustrative Limits 9

3.4 One-Loop Corrected Bulk-Boundary Green Functions 10

3.4.1 Numerical Results 11

4 Absence of Friedel Oscillations in Electron Stars 12

4.1 Electron Star Background 13

4.2 Electron Star Static Correlation Functions: Shear Channel 14

4.2.1 Static Limit 14

$\begin{array}{lll}4.3 & \text { Polarized Electron Star } & 16\end{array}$

$\begin{array}{ll}\text { 4.3.1 Lack of Friedel Oscillations } & 17\end{array}$

$\begin{array}{llr}5 & \text { Discussion } & 18\end{array}$

A Perturbative Polarization Tensor $\quad 19$

A.1 Static Limit 20

B Holographic Relative Polarization Tensor $\quad 21$

B.1 High Confinement Scale and Non-Relativistic Limit 21

C Flat Space QED Polarization Tensor 23

$\begin{array}{ll}\text { References } & 24\end{array}$

\section{Introduction}

Recent years have seen a number of holographic constructions aimed at modeling fermions at finite density in $2+1$ dimensions (see [1-3] for recent reviews). A primary motivation has been to find a suitable gravity dual for non-Fermi liquids, that is 
metals whose low energy dynamics differ from those of the usual Landau-Fermi liquid. In conventional metals the robustness of Fermi liquid theory has been argued in terms of an IR stable Gaussian fixed point of the renormalization group governing the lowenergy excitations of charged fermions about a Fermi surface in $3+1$ dimensions $[4,5]$. If, on the other hand, the physics of the conduction electrons is essentially confined to two spatial dimensions this no longer applies. The low energy excitations of charged fermions about a Fermi surface in $2+1$ dimensions are strongly coupled and perturbative field theory cannot be relied upon [6-8].

In this context, gauge/gravity duality may provide useful new insights and considerable effort has been put into developing holographic duals of strongly coupled fermion dynamics in $2+1$ dimensions [9-27]. An important step is to demonstrate how a Fermi surface may be encoded in a holographic geometry. One approach is to couple a probe fermion to the bulk gravitational sector and compute the resulting spectral density [9-12, 21]. This has led to many interesting results, including signals of Fermi surfaces, Fermi liquids, and even non-Fermi liquid behavior. Interesting characteristics of the probe fermion arise because the spacetime geometry and other bulk fields serve to provide a strongly coupled gapless sector to which the probe is weakly coupled [15]. The probe fermion results are, however, rather indirect from the point of view of the holographic background which, by definition, does not know about the presence of probes.

In the present paper, we instead look for signals of Fermi surfaces in static susceptibilities, such as a current-current correlator at finite momentum and zero frequency [28]. Such correlation functions are inherent to the holographic setup and the relevant gauge couplings are determined by the same bulk Lagrangian as the gravitational background itself. Specifically, we expect to see non-analytic features, so called Friedel oscillations, at twice the Fermi momentum in zero temperature static current-current correlation functions when there are a discrete set of Fermi surfaces. Friedel oscillations are expected whenever there is a sharp Fermi surface, and do not rely upon the assumptions of Fermi liquid theory.

The holographic models that we consider have three key ingredients in common. One is that the gravitational dual spacetime is asymptotically AdS, which corresponds to an underlying conformal symmetry in the boundary field theory. Relaxing the condition of conformal symmetry is of considerable interest but this will not be pursued here. Another shared ingredient is a U(1) electric flux threading the asymptotic AdS region, which is the holographic manifestation of a non-vanishing chemical potential and charge density in the boundary theory. The finite charge densities at zero temperature indicate that the dual field theory also possesses Fermi surfaces by a generalized Luttinger's theorem [29]. Finally, we have chosen to focus on models where the asymptotic electric flux is sourced by a finite density of charged fermions inside the bulk rather than emanating from behind the event horizon of a charged black hole. 
One such system is an electron star, first considered in $[14,17]$. There the approach is to increase the charge density of bulk fermions, such that they may be considered as a fluid of degenerate fermions. In this limit, there is a continuum of bulk Fermi surfaces, distributed radially. Further, in a certain parameter regime, one can ignore the effect of gravity on the fluid's local equation of state. By setting up the corresponding Oppenheimer-Volkov equations it is possible to numerically determine the spacetime's geometry. Electron stars are non-singular geometries possessing Lifshitz scaling regimes in the deep interior. One of the initial hopes for electron stars was that, by working with macroscopic bulk charge densities, they would make fermionic properties visible at the classical level. Indeed, in [21, 23], it was shown, at the probe fermion level, that electron stars support many discrete Fermi surfaces which satisfy a Luttinger count. On the other hand, as discussed in [19], most of the bulk Fermi surfaces do not contribute to de Haas-Van Alphen oscillations in a background magnetic field. These are dominated by a single Fermi surface while the contributions from the remaining local Fermi surfaces in the bulk fluid wash out. We will find similar smearing when we look for Friedel oscillations in electron star backgrounds except in this case the signal is completely smeared out.

Another model for holographic Fermi surfaces, which we will refer to as an "AdS hard wall," was introduced in [25]. This model was intended as a toy model where a finite number of discrete Fermi surfaces could be made manifest. Interpreting the difficulties encountered with extremal black holes and electron stars as a signal of too many IR degrees of freedom, [25] instead works within a confining dual gauge theory, achieved via a truncation of an AdS geometry by introducing "hard wall" boundary conditions at a finite value of the radial variable. The profile of a bulk gauge field in the presence of a filled bulk Fermi surface is solved for numerically, neglecting any gravitational back reaction, and then the effect of the resulting gauge field on the bulk fermions is solved for. These steps are iterated towards a self-consistent configuration of the fermions and gauge field. A Luttinger count indicated that the resulting theory could describe either Fermi or non-Fermi liquids depending on the gauge field's boundary conditions at the hard wall [25]. Morally, this configuration is similar to that of an electron star with a non-vanishing charge density carried by bulk fermions, but differs crucially in the number of IR degrees of freedom. Also, the character of bulk Fermi surfaces is very different than in the electron star geometry. In the hard wall construction, a bulk Fermi surface is not a local concept, but instead each Fermi surface is assigned a radial mode number. The non-locality in the bulk Fermi surface leads to a certain factorization in the way radial and boundary information is encoded, implying that the AdS space outside the hard wall acts more or less like a finite size box, as discussed in [25].

Our understanding of the application of holography to fermionic systems at finite density is in its infancy. The electron star and hard wall geometries provide two systems through which we may develop our intuition. By contrasting the two 
backgrounds we compare the effects of bulk Fermi surfaces which are local vs. nonlocal and continuous vs. discrete. In order to see signatures of Fermi surfaces in current correlation functions, which involve local bosonic operators, it is necessary to include the effects of bulk fermionic loops. This is because fermionic operators do not have expectation values. The primary result of this paper will be to demonstrate that bulk Fermi surfaces in an AdS hard wall geometry can induce boundary Friedel oscillations, while a similar calculation for electron stars shows that the oscillations are washed out due to the continuum of bulk Fermi surfaces. These results suggest that discrete bulk Fermi surfaces which are non-local in the radial direction induce sharp, discrete boundary Fermi surfaces.

The paper is outlined as follows. We begin with a review of Friedel oscillations in a $2+1$ dimensional perturbative system in Section 2. In Section 3 we briefly discuss the AdS hard wall background introduced in [25] and then we identify the source of Friedel oscillations. In Section 4 we set up an analogous calculation in an electron star geometry but find that there are no boundary Friedel oscillations due to the continuum of bulk Fermi surfaces. Finally, we conclude with a discussion in Section 5. In order to streamline the presentation the technical details are relegated to appendices when possible.

\section{Friedel Oscillations}

In systems with sharp Fermi surfaces, zero temperature, static response functions display spatial oscillatory behavior, called Friedel oscillations. ${ }^{1}$ Heuristically, this may be understood as follows: Having a sharp, non-analytic, cut in the quasi-particle density of states leads to non-analytic features in momentum space static response functions. Upon Fourier transformation, these non-analyticities give rise to oscillatory spatial features at wavenumber $2 k_{f} .{ }^{2}$ Such oscillations occur in all known models with sharp Fermi surfaces, for essentially any static response function considered (i.e. density-density, spin-spin, etc.). Heuristically, the character of response functions changes depending on whether the exchanged momentum "fits" in the Fermi sphere. In this section we will outline how this works for $2+1$ dimensional non-relativistic fermions, leaving the details for Appendix A. Understanding the perturbative calculation will be useful to help set notation and because it has close parallels with the

\footnotetext{
${ }^{1}$ Strictly speaking Friedel oscillations refer to spatial oscillations occurring in charge density perturbations. In this article we will use the term more generally to refer to spatial oscillations in generic transport coefficients due to a sharp Fermi surface.

${ }^{2} \mathrm{~A}$ simple example is given by the Fourier transform of a one-dimensional sharp bump function in momentum space of width $w$ and unit height,
}

$$
F . T .[B(k)]=\sin (w x) / w .
$$


holographic calculation. Similar effects are expected in non-Fermi liquids since it is the sharpness of the Fermi surface that matters rather than the detailed dynamics.

\subsection{Perturbative Friedel Oscillations in $2+1$ Dimensions}

Friedel oscillations are easily visible in the density-density polarization (i.e. susceptibility), denoted by П. For simplicity we will focus on a system of spinless fermions with a Fermi momentum, $k_{f}$. For weakly coupled fermions this is textbook material, see for example [30, 31].

In a unitary system we can use a Lehmann representation to capture the full frequency dependence of two point functions. The time ordered Green's function for free fermions in a translationally and rotationally invariant system takes the form

$$
\mathcal{G}(k, \omega)=\frac{\theta\left(|k|-k_{f}\right)}{\omega-E_{k}+i \eta}+\frac{\theta\left(k_{f}-|k|\right)}{\omega-E_{k}-i \eta},
$$

in $(\omega, k)$ space. Using the fermion's Green's function, an application of Kubo's formula indicates that the leading contribution to the polarization is

$$
\Pi(q, \nu)=-i e^{2} \int \frac{d \omega d \vec{k}}{(2 \pi)^{D+1}} \mathcal{G}(k, \omega) \mathcal{G}(k+q, \omega+\nu) .
$$

In the static limit the polarization tensor is real

$$
\begin{aligned}
& \operatorname{Im} \Pi(q, 0)=0, \\
& \operatorname{Re} \Pi(q, 0)=-\frac{e^{2}}{2 \pi^{2}} \mathcal{P} \int d k d \theta \theta\left(k_{f}-k\right) \frac{k}{E_{k+q}-E_{k}} .
\end{aligned}
$$

For spherical Fermi surfaces the integrand is singular when $q=2 k_{f}$. Even without knowing the precise form of the dispersion relation, the polarization tensor is nonanalytic in momentum space and thus has oscillatory behavior in position space.

For concreteness, consider non-relativistic free fermions with $E_{k}=\frac{k^{2}}{2 m}$. In this case (2.5) reduces to

$$
\begin{aligned}
\Pi(q, 0) & =-\frac{e^{2} m}{2 \pi^{2} q} \mathcal{P} \int d k d \theta \theta\left(k_{f}-k\right) \frac{k}{q / 2+k \cos (\theta)} \\
& =-\frac{e^{2} m}{2 \pi}\left(1-\sqrt{1-\left(\frac{2 k_{f}}{q}\right)^{2}} \theta\left(q-2 k_{f}\right)\right) .
\end{aligned}
$$

The Kubo formula relates the charge density susceptibility to the polarization tensor

$$
\chi(q) \equiv\langle\rho(-q) \rho(q)\rangle_{R}=-\Pi_{R}(q)=-\Pi(q) .
$$

In the last equality we have used the fact that the static polarization tensor is real to equate its time-ordered and retarded incarnations. In momentum space the nonanalyticity at $q=2 k_{f}$ is clearly visible in Figure 1 . 


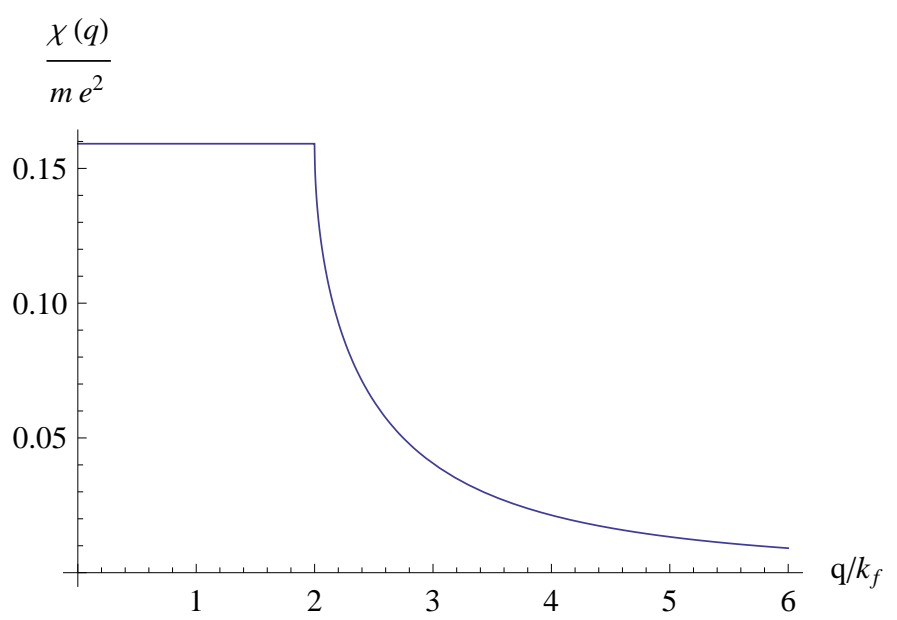

Figure 1. The non-relativistic static susceptibility, $\chi(q)=-\Pi(q)$, in $2+1$ dimensions for spinless fermions of mass $m$ and charge $e$.

\section{Friedel Oscillations in an AdS Hard Wall Geometry}

As mentioned in Section 1, a number of holographic models have been introduced to describe Fermi surfaces at strong coupling. To reduce the number of IR degrees of freedom, the author of [25] proposed to work with confining boundary field theories. As is familiar from using holography to study QCD, we can model confinement with a hard wall placed in the bulk [32]. Although this model does not include the effects of a bulk Fermi surface on the metric degrees of freedom, the Fermi surface does back react onto the gauge field's profile. Therefore, in this model one can study the effects of a bulk Fermi surface outside of a probe limit on fermionic or current correlators, but not on stress tensor correlators. Here we will focus on current correlators and show that they display boundary Friedel oscillations.

\subsection{Preliminaries}

Following [25], we begin with pure $\mathrm{AdS}_{4}$,

$$
d s^{2}=\frac{L^{2}}{z^{2}}\left(-d t^{2}+d z^{2}+d \vec{x}^{2}\right) .
$$

In order to control the number of fields in the dual field theory's IR limit, one introduces a hard wall cutoff, $z_{m}$. We can imagine that the cutoff is an approximation to some more interesting geometric effect [33]. On this background geometry we place both fermions and gauge fields governed by the standard action

$$
S=\int d^{4} x \sqrt{-g}\left[\frac{1}{4 e^{2}} F^{2}+\bar{\Psi}(i \not D+m) \Psi\right] .
$$

The covariant derivative is $D_{\mu}=\partial_{\mu}+\frac{1}{4} \omega_{a b \mu} \Gamma^{a b}-i q A_{\mu}$. We will be interested in constructing the background gauge field profiles and understanding linear response 
theory in the gauge sector on large length scales. For these tasks it is sufficient to integrate out the fermions and use the resulting low energy effective action

$$
S=\int d^{4} x \sqrt{-g} \frac{1}{4 e^{2}} F^{2}+\operatorname{Tr} \ln [i \not D+m] .
$$

In addition to the differential equations coming from Eq. (3.2), one must also specify boundary conditions at the hard wall. Because the geometry is regular, it is natural to impose self-adjoint boundary conditions on the fermions [25]. Such boundary conditions are quite strong, however, ruling out any dissipation in the fermionic sector in the large $N$ limit.

With the boundary conditions in place, we seek to fill fermion states up to the ground state energy as well as determining the self-consistent gauge field profile. In practice this may be done through an iterative process. As discussed in [25], Neumann boundary conditions for $A_{0}$ at the hard wall, are a necessary condition to have a boundary Fermi liquid in this set up. Any other self-adjoint boundary condition leads to non-Fermi liquid behavior. The resulting background consists of a filled sea of fermion single particle wave functions, each indexed by boundary momentum as well as a radial mode number. In addition one must include the backreaction of the Fermi sea on the gauge field. For chemical potentials above the gap, the system has compressible [34] gapless excitations and a sharp Fermi surface.

It is useful to consider the time ordered Green's function of these normalizable fermions. Using a Lehmann representation we have

$$
G\left(\omega, k, z, z^{\prime}\right)=\sum_{\ell \neq 0}\left(\frac{1}{\omega-E_{\ell}(k)+i \eta \operatorname{sign}\left(E_{\ell}(k)\right)}\right) \chi_{\ell, k}(z) \chi_{\ell, k}^{\dagger}\left(z^{\prime}\right),
$$

where the wave functions and energies are those numerically determined when solving for the background, and $E_{\ell}(k)$ is the eigenvalue of the Dirac Hamiltonian with spatial momentum $k$ and radial mode number $\ell$.

\subsection{Mean Field Susceptibility}

Here we turn to the problem of linear response theory in hard wall AdS in the radial gauge, $A_{z}=0$. The (static) classical wave equation for the potential is

$$
\left(\partial_{z}^{2}-k_{x}^{2}\right) A_{0}\left(k_{x}, z\right)=0 .
$$

For definiteness we have taken the momentum to be in the $x$ direction and we focus on a gauge invariant sector of perturbations $A_{0}\left(k_{x}\right)$. Since the hard wall is a regular point in the geometry, it is natural to impose the same boundary conditions that are used in constructing the background. We can find the classical bulk to boundary Green's function (i.e. the solution which goes to one at the boundary) analytically.

$$
\begin{aligned}
G_{0}^{B \partial}\left(k_{x}, z\right) & =\cosh \left(k_{x} z\right)-\tanh \left(k_{x} z_{m}\right) \sinh \left(k_{x} z\right), \\
\rightarrow\left\langle\rho\left(-k_{x}\right) \rho\left(k_{x}\right)\right\rangle_{0} & =-k_{x} \tanh \left(k_{x} z_{m}\right)
\end{aligned}
$$


As expected, the classical contribution to the susceptibility does not know about the Fermi surface. We must go away from mean field theory and consider virtual particle-hole exchanges.

\subsection{Polarization Corrections}

In order to proceed, let us imagine integrating out the fermions in the bulk. Graphically, the first correction to the gauge field's effective action comes from a bubble diagram with fermions in the loop. ${ }^{3}$ If we expand out the fermion's functional determinant to second order in gauge fields, we have a term of the form

$$
S_{P o l}=\frac{e^{2}}{2} \int d z d z^{\prime} d \vec{x} A_{\mu}(z,-\vec{k}) \Pi^{\mu \nu}\left(z, z^{\prime}, \vec{k}\right) A_{\nu}\left(z^{\prime}, \vec{k}\right)
$$

The one loop vacuum polarization tensor is written

$$
\Pi^{\mu \nu}\left(z, z^{\prime}, \nu, \vec{k}\right)=-\int \frac{d \omega d^{2} p}{(2 \pi)^{3}} \operatorname{Tr}\left[M^{\mu} G\left(\omega, p, z, z^{\prime}\right) M^{\nu} G\left(\omega+\nu, k+p, z^{\prime}, z\right)\right],
$$

where $M^{\mu}=-q \Gamma^{0} \Gamma^{\mu}$. The reason that $M^{\mu} \neq-q \Gamma^{\mu}$ is that we have chosen to work with the Green's function involving $\chi_{k, \ell}(z) \chi_{k, \ell}^{\dagger}\left(z^{\prime}\right)$ rather than $\chi_{k, \ell}(z) \bar{\chi}_{k, \ell}\left(z^{\prime}\right)$. In what follows we will limit our discussion to the static polarization tensor, $\Pi^{\mu \nu}\left(z, z^{\prime}, 0, \vec{p}\right) \equiv$ $\Pi^{\mu \nu}\left(z, z^{\prime}, \vec{p}\right)$. In the background geometry there are three parameters, namely $\mu, q, e$, and only two of them are fixed by the classical geometry [25]. The relative size of the loop correction (3.8) is determined by the third one. ${ }^{4}$

In general, evaluating the vacuum polarization in curved space is a rather difficult problem. There are the usual ultraviolet divergences occurring due to large $\ell$ and $k$. Also, one might worry about the cornucopia of fields predicted by string theory predict in an eventual top down approach. As discussed in [30], we can split the vacuum polarization into two pieces: the (uninteresting) contributions present in vacuum and the relative polarization due to a chemical potential,

$$
\Pi^{\mu \nu}=\left.\Pi^{\mu \nu}\right|_{v a c}+\Pi_{r e l}^{\mu \nu}
$$

If the chemical potential is not too high, we can assume that the vacuum part only contributes to the ultraviolet renormalization of parameters in Eq. (3.2), and is not relevant to the long wavelength physics associated with Friedel oscillations.

\footnotetext{
${ }^{3}$ At zero momentum the effective action for $A_{0}$ also has a tadpole contribution analogous to the one used to determine the gauge field's background profile. This term does not affect our results below.

${ }^{4}$ To anticipate the notation used in Section 4 below, we have rescaled the gauge field $A_{\mu} \rightarrow \frac{e L}{\kappa} A_{\mu}$, as in Eq. (4.2), with $L$ the curvature radius, and $\kappa$ the Newton constant. With this normalization and with the metric as in Eq. (3.1), the background action has an overall factor of $\frac{L^{2}}{\kappa^{2}}$, which we have factored out in (3.8).
} 
If we denote the vacuum time-ordered bulk fermion Green's function by $G^{0}$, the relative polarization tensor may be written as,

$$
\begin{array}{r}
\Pi_{r e l}^{\mu \nu}\left(z, z^{\prime}, \vec{k}\right)=-\int \frac{d \omega d^{2} p}{(2 \pi)^{3}} \operatorname{Tr}\left[M^{\mu} G\left(\omega, \vec{p}, z, z^{\prime}\right) M^{\nu} G\left(\omega, \vec{k}+\vec{p}, z^{\prime}, z\right)\right] \\
-\operatorname{Tr}\left[M^{\mu} G^{0}\left(\omega, \vec{p}, z, z^{\prime}\right) M^{\nu} G^{0}\left(\omega, \vec{k}+\vec{p}, z^{\prime}, z\right)\right] .
\end{array}
$$

We analyze the relative polarization in Appendix B. The full result is in general quite involved but fortunately we do not need it in full detail. Let us list some important features. First of all, the relative polarization is finite in the bulk UV. This is analogous to the usual finite temperature field theory statement that the only UV divergences are vacuum divergences. Second, the integrand cleanly splits into terms unrelated to the Fermi surface which are analytic in the external momenta near $2 k_{f}$ and contributions that have singular points at $k=2 k_{f}$. These singularities do not lead to actual divergences in the polarization but they are the source of bulk Friedel oscillations in much the same way as in the non-relativistic example in Section 2.1.

\subsubsection{Illustrative Limits}

As discussed in detail in Appendix B.1, there is a limit where the relative polarization radically simplifies and one can obtain an (almost) analytic expression. If we consider a high confinement scale (small $z_{m}$ ) then all contributions to the loop diagram with non-zero relative radial momentum ${ }^{5}$ are suppressed by factors of $z_{m}$ and may be dropped. The integrand then only receives contributions from near a Fermi surface.

We can make a stronger statement if we take a non-relativistic limit and tune the chemical potential towards the fermion's bulk gap energy, leading to a Fermi surface of small volume. For simplicity, consider a background with a single Fermi surface, of mode number 1. As seen in Figure 2, the numerically determined dispersion relation is very well approximated by a quadratic form near the Fermi surface

$$
E_{1}(k) \sim a_{1}+b_{1} k^{2}
$$

Assuming this functional form, the vacuum polarization is well approximated by

$$
\begin{aligned}
\Pi_{r e l}^{\mu \nu}\left(z, z^{\prime}, \vec{k}\right)= & -\lambda \operatorname{Tr}\left(M^{\mu} \chi_{1,0}(z) \chi_{1,0}^{\dagger}\left(z^{\prime}\right) M^{\nu} \chi_{1, \vec{k}}\left(z^{\prime}\right) \chi_{1, \vec{k}}^{\dagger}(z)\right) \\
& \times\left(1-\sqrt{1-\left(\frac{2 k_{f}}{|\vec{k}|}\right)^{2}} \theta\left(|\vec{k}|-2 k_{f}\right)\right),
\end{aligned}
$$

as discussed in Appendix B.1. We have introduced an effective expansion parameter, $\lambda \equiv \frac{1}{4 \pi b_{1}}$ which encodes the shape of the spectrum. The source of bulk (and

\footnotetext{
${ }^{5}$ More precisely contributions where the fermion propagators have different radial mode numbers.
} 


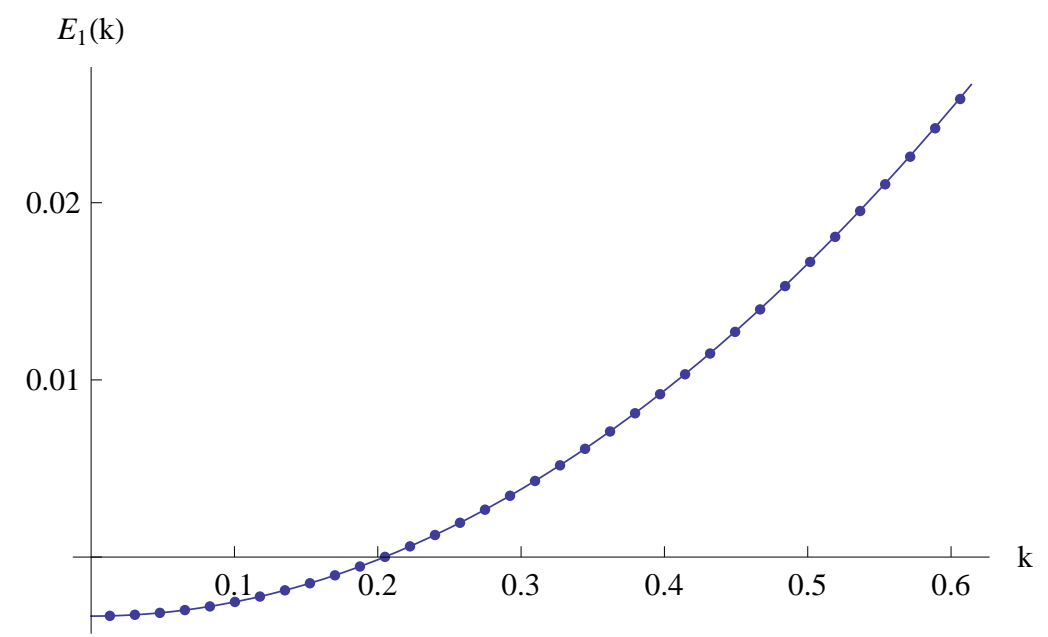

Figure 2. The energy dispersion relation for the $\ell=1$ family of eigenvalues for $\left(m, q, e, \mu, z_{m}\right)=(1,1, \sqrt{3}, 6.287, .5)$ with $k_{f} \sim .20$ as well as the best fit curve $E_{1} \sim$ $-.003+.8 k^{2}$.

boundary) Friedel oscillation is now explicit in this non-relativistic limit. Interestingly, there is an important difference compared to the perturbative calculation in Section 2.1 in that the polarization is a non-diagonal tensor. This amounts to nonvanishing mixed density-current correlation functions in the holographic dual.

\subsection{One-Loop Corrected Bulk-Boundary Green Functions}

Returning to the gauge invariant sector, it is straightforward to set up of the diffeointegral equations for the loop corrected gauge field equations of motion,

$$
\left(\partial_{z}^{2}-k_{x}^{2}\right) A_{0}\left(k_{x}, z\right)=-e^{2} \int d z^{\prime}\left[\Pi_{r e l}^{00}\left(z, z^{\prime}, k_{x}\right) A_{0}\left(k_{x}, z^{\prime}\right)+\Pi_{r e l}^{0 x}\left(z, z^{\prime}, k_{x}\right) A_{x}\left(k_{x}, z^{\prime}\right)\right] .
$$

The absence of metric factors on the left-hand side is a peculiarity of the gauge field equation of motion in $\mathrm{AdS}_{4}$. We will work in a background where the gauge nontrivial part of $A_{x}$ vanishes such that we can drop the contribution from the second term on the right hand side of (3.14).

If we have a high confinement scale and a small Fermi surface volume, the diffeointegral equation can be solved perturbatively in $\lambda$ for the bulk to boundary Green's functions

$$
G^{B \partial}\left(k_{x}, z\right)=G_{0}^{B \partial}\left(k_{x}, z\right)+\lambda G_{1}^{B \partial}\left(k_{x}, z\right)+\ldots \quad(t-\text { component }) .
$$

This expansion should be valid as long as loop effects are small. Expanding (3.14) we find, 

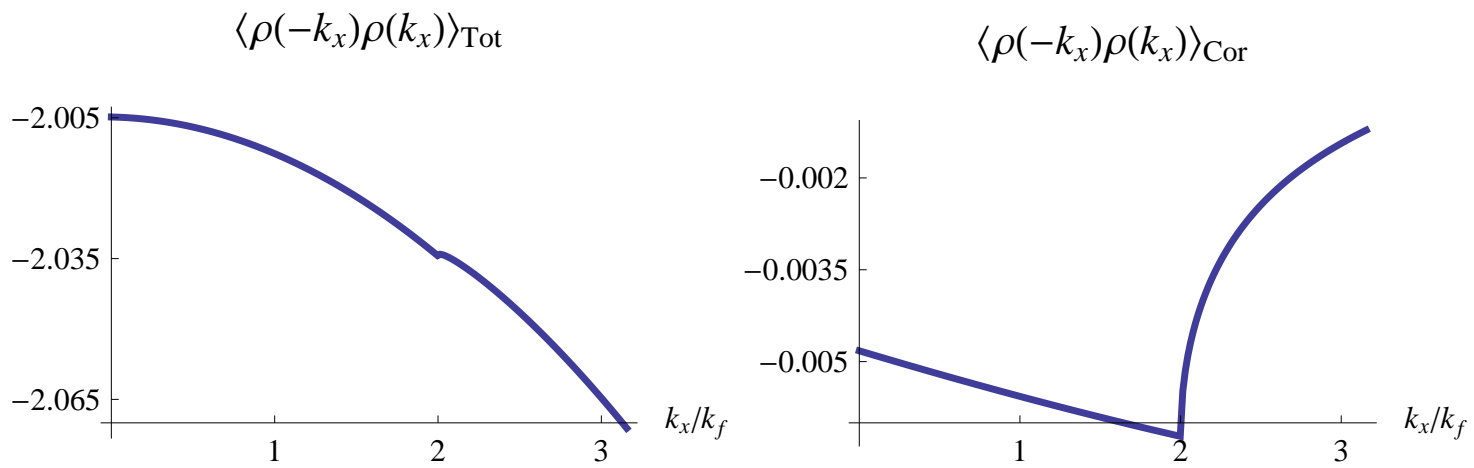

Figure 3. Correlation functions for $\left(m, q, e, \mu, z_{m}\right)=(1,1, \sqrt{3}, 6.287, .5)$ with $k_{f} \sim .20$ in a hard wall geometry. On the left, the full density-density correlation function computed by including the one-loop effect induced by the polarization term (3.8). On the right, only the one-loop contribution to the density-density correlator is plotted.

$$
\begin{aligned}
& 0=\left(\partial_{z}^{2}-k_{x}^{2}\right) G_{0}^{B \partial}\left(k_{x}, z\right), \\
& 0=\left(\partial_{z}^{2}-k_{x}^{2}\right) G_{1}^{B \partial}\left(k_{x}, z\right)+\frac{e^{2}}{\lambda} \int d z^{\prime} \Pi_{r e l}^{00}\left(z, z^{\prime}, k_{x}\right) G_{0}^{B \partial}\left(k_{x}, z^{\prime}\right) .
\end{aligned}
$$

The leading contribution, $G_{0}^{B \partial}$, was determined in Eq. (3.6). Since the leading contribution satisfies the physical boundary condition, $G_{0}^{B \partial}(k, 0)=1$, the corrections must satisfy Dirichlet conditions near $z \sim 0$.

\subsubsection{Numerical Results}

The perturbative equations (3.17) can be solved numerically and using the AdS/CFT dictionary it is then straightforward to translate the bulk to boundary Green's functions into correlation functions in the boundary theory. Figure 3 shows the resulting density-density susceptibility with and without the uninteresting classical contribution. At the $\mathcal{O}(1 / N)$ level we clearly see a non-analyticity at $k=2 k_{f}$, the hallmark of a sharp Fermi surface. The analysis in this section is performed for Neumann hard wall conditions. It would be interesting to extend the analysis to other boundary conditions. Intuition from field theory suggests that Friedel oscillations should still be visible whenever there is a sharp Fermi surface regardless of the stability of the quasi-particles at the Fermi surface.

The loop corrections involve the effective loop parameter, $\frac{L}{\kappa}$, encoding $1 / N$ corrections which did not play a role in when determining the classical gauge field background. The value of the loop parameter does not affect the position of the nonanalyticity in (3.14) and we have set it to one in order to maximize the amplitude of boundary Friedel oscillations in our numerical results.

This example illustrates several important points relevant when trying to construct boundary Fermi surfaces from Fermi surfaces in the bulk. Firstly, even when 
the role of the bulk Fermi surface is manifest in the construction of the background profiles, classically computed linear response susceptibilities typically do not reveal the surface. As in perturbative descriptions, one is looking for evidence of particle hole pairs in bosonic observables, which are only present in loops (though the loops are now in the bulk). As discussed in [25], Luttinger's theorem fixes the boundary value of $k_{f}$ to be the same as the bulk value. The amplitude of Friedel oscillations is thus $N$ dependent, but not the wave vector at which they occur.

Secondly, the presence of a bulk Fermi surface does not guarantee a clear signal in the boundary theory. To be visible, effects of bulk Fermi surfaces must act coherently across the radial direction. For example, when we compute corrected SchwingerDyson equations, there are convolution integrals in the radial direction. In order to prevent the convolution integrals from washing out the effects of a bulk Fermi surface, the spectrum of radial momenta must be gapped. In the model of [25], it is the confinement scale which introduces the gap.

Thirdly, even if one can neglect smearing due to convolutions, it is necessary to have factorization between the dependence on the boundary momenta and the radial positions. In this hard wall example this feature is visible in the form of Eq. (3.13) (or more generally in Eq. (B.3)). The relevant singularities in the integrand are only functions of boundary momenta. In the hard wall example it is the radial non-locality of the Fermi surface which makes this happen.

In the next section we will discuss electron star geometries which also have a finite density of fermions in the bulk. However, this geometry differs in two crucial ways from the hard wall model. The spectrum of radial momenta is not gapped and the bulk fermionic features (such as the Fermi momenta) are local functions of the radial positions.

\section{Absence of Friedel Oscillations in Electron Stars}

Another holographic model for the physics of finite density fermions are electron stars $[14,17]$. The basic elements include a fluid of charged electrons treated as an ideal fluid of non-interacting particles at zero temperature, i.e. a Thomas-Fermi approximation. The star geometry carries charge more efficiently than an extremal black hole and hence it is thermodynamically preferred at zero temperature. In the deep interior the gravitational solution goes over to a Lifshitz scaling solution. In [26, 27], the finite temperature extension of this geometry was found. As for holographic superconductors, at low temperatures it is thermodynamically preferred for the charged black holes to expel charged matter. The preferred low temperature geometry consists of a charged black hole surrounded by a fermion fluid of the same sign charge. Above a critical temperature there is a third order phase transition from an electron cloud over to an AdS-RN black hole. We will refer to the zero temperature geometry as an electron star and to its finite temperature generalization as an electron 
cloud. Although both the hard wall fermion background and the electron star have bulk Fermi surfaces there are important differences, which will allow us to test the lessons mentioned in Section 3.4.1. Ultimately, we find that bulk Friedel oscillations will wash out, leaving no sharp signature in the boundary current-current correlation function in the electron star.

\subsection{Electron Star Background}

The electron star geometry is given by ${ }^{6}$

$$
\begin{aligned}
d s^{2} & =\frac{L^{2}}{z^{2}}\left(-f(z) d t^{2}+g(z) d z^{2}+d \vec{x}^{2}\right) \\
A & =\frac{e L}{\kappa} h(z) d t
\end{aligned}
$$

where $e$ is the fermion charge, $L$ is the curvature radius, and $\kappa$ is Newton's constant. In each local patch there is a degenerate fluid of free fermions modeled as an ideal fluid described with velocity, pressure, energy density, and charge density, $\left(u_{\mu}, \hat{p}, \hat{\rho}, \hat{\sigma}\right){ }^{7}$ In the scaling regime the geometry takes the form

$$
f \sim \frac{1}{z^{2(s-1)}}, \quad g \sim g_{\infty}, \quad \text { and } \quad h \sim \frac{h_{\infty}}{z^{s}} .
$$

In the rest of the spacetime the background profiles are non-singular functions of the radial coordinate interpolating between an interior Lifshitz region and a nearboundary AdS region.

One of the initial motivations for the electron star model was to make the fermionic features more dominant by working at finite fermion densities. Indeed, in [19], it was shown that the thermodynamic potential displays de Haas-van Alphen oscillations due to a bulk Fermi surface. However it is also clear that the fermionic character of the electron star geometry is quite different than in the hard wall model of Section 3. Most importantly, there is a continuum of Fermi surfaces in the ThomasFermi approximation. At each radial position there is an effective local chemical potential

$$
\hat{\mu}=\frac{z h}{\sqrt{f}} .
$$

\footnotetext{
${ }^{6}$ Note that we have slightly redefined the metric functions $f$ and $g$ relative to [17]. We will also denote the dynamical critical exponent by $s$ rather than the usual $z$ to avoid confusion with the radial coordinate.

${ }^{7}$ The fluid variables are rescaled as in [17],

$$
p=\frac{1}{L^{2} \kappa^{2}} \hat{p}, \quad \rho=\frac{1}{L^{2} \kappa^{2}} \hat{\rho}, \quad \sigma=\frac{1}{e L^{2} \kappa} \hat{\sigma} .
$$

Furthermore, the mass of the fermions and the constant proportional to the spin in the fermion equation of state are rescaled according to $m=\frac{e}{\kappa} \hat{m}, \beta=\frac{\kappa^{2}}{e^{4} L^{2}} \hat{\beta}$.
} 
A second important difference is the electron star's lack of confinement and a relative surplus of infrared degrees of freedom. The emergence of a Lifshitz scaling region in the geometry's interior implies that there is no gap in the allowed radial momenta.

In the rest of this section we discuss Friedel oscillations in the electron cloud geometry. We work with the finite temperature electron cloud geometry rather than the zero temperature electron star. This is purely for convenience when carrying out numerical calculations. Since the electron star geometry, including the Lifshitz scaling region, is recovered when the temperature of an electron cloud geometry is taken to be very low compared to the scale set by the chemical potential, we can expect to recover electron star correlation functions from the ones obtained from electron clouds in the low temperature limit. Furthermore, as the temperature of an electron cloud geometry is given by the Hawking temperature of the black hole horizon below the electron cloud while treating the electron cloud itself as a zero temperature fluid [26], any bulk "medium" effects should be visible even at finite temperature.

As a second technical simplification we will not look for Friedel oscillations in the sound channel, the natural generalization of Section 3.2. Instead, we will use the fact that any channel is expected to display Friedel oscillations in the presence of a sharp Fermi surface and work with the much simpler shear channel.

\subsection{Electron Star Static Correlation Functions: Shear Channel}

Not surprisingly, analyzing the linear response theory for electron stars and electron clouds is considerably more complicated than for the hard wall. If we consider a general perturbation with boundary frequency and momentum $\left(\omega, k_{y}\right)$, we can classify the allowed perturbations according to their behavior under the residual $\mathbb{Z}_{2}$ symmetry, $(x \rightarrow-x)$ as discussed in $[35,36]$. In asymptotically $\mathrm{AdS}_{4}$, there are two families of perturbations in the presence of the fluid in the radial gauge $\delta A_{z}=0, \delta g_{z \mu}=0$

$$
\begin{aligned}
\text { odd : } & \left(\delta g_{x y}, \delta g_{x t}, \delta A_{x}, \delta u_{x}\right) \\
\text { even : } & \left(\delta g_{x x}, \delta g_{y y}, \delta g_{t t}, \delta g_{t y}, \delta A_{y}, \delta A_{t}, \delta u_{t}, \delta u_{z}, \delta u_{y}\right) .
\end{aligned}
$$

The family of odd perturbations give rise to shear modes while the even family corresponds to sound modes in the dual field theory. Here we will focus on the shear channel because it includes a smaller set of fields.

\subsubsection{Static Limit}

In general, the gravitational response theory is complicated by the fact that there are gravitational gauge symmetries in the form of diffeomorphisms. In the static limit there is a simplification and we only need to retain $\delta g^{t}{ }_{x}, \delta u_{x}$, and $\delta A_{x}$. It is straightforward to set up the classical fluctuation equations describing gauge invariant shear 
modes in the $\omega \rightarrow 0$ limit,

$$
\begin{aligned}
& 0=\left(\delta g_{x}^{t}\right)^{\prime \prime}+\frac{(g \hat{\mu} \hat{\sigma}-4)}{2 z}\left(\delta g_{x}^{t}\right)^{\prime}-g k_{y}^{2} \delta g_{x}^{t}-\frac{2 \sqrt{f} g \hat{\sigma}}{z} \hat{\mu} \delta u_{x}+2 z^{2} h^{\prime}\left(\delta A_{x}\right)^{\prime}, \\
& 0=\left(\delta A_{x}\right)^{\prime \prime}-g k_{y}^{2} \delta A_{x}+\frac{h^{\prime}}{f}\left(\delta g_{x}^{t}\right)^{\prime}-\left(\frac{g^{\prime}}{g}+\frac{g \hat{\mu} \hat{\sigma}}{2 z}\right)\left(\delta A_{x}\right)^{\prime}+\frac{g \hat{\sigma}}{z^{2}} \delta u_{x} .
\end{aligned}
$$

In addition to the equations of motion, the divergence of the stress-energy tensor fixes

$$
\delta A_{x}=-\hat{\mu} \delta u_{x}
$$

For an electron cloud geometry, it is straightforward to compute Euclidean boundary susceptibilities by imposing regularity conditions at the horizon. As in the nonrelativistic example we find a real static susceptibility and hence the Euclidean result equals its Lorentzian counterpart.

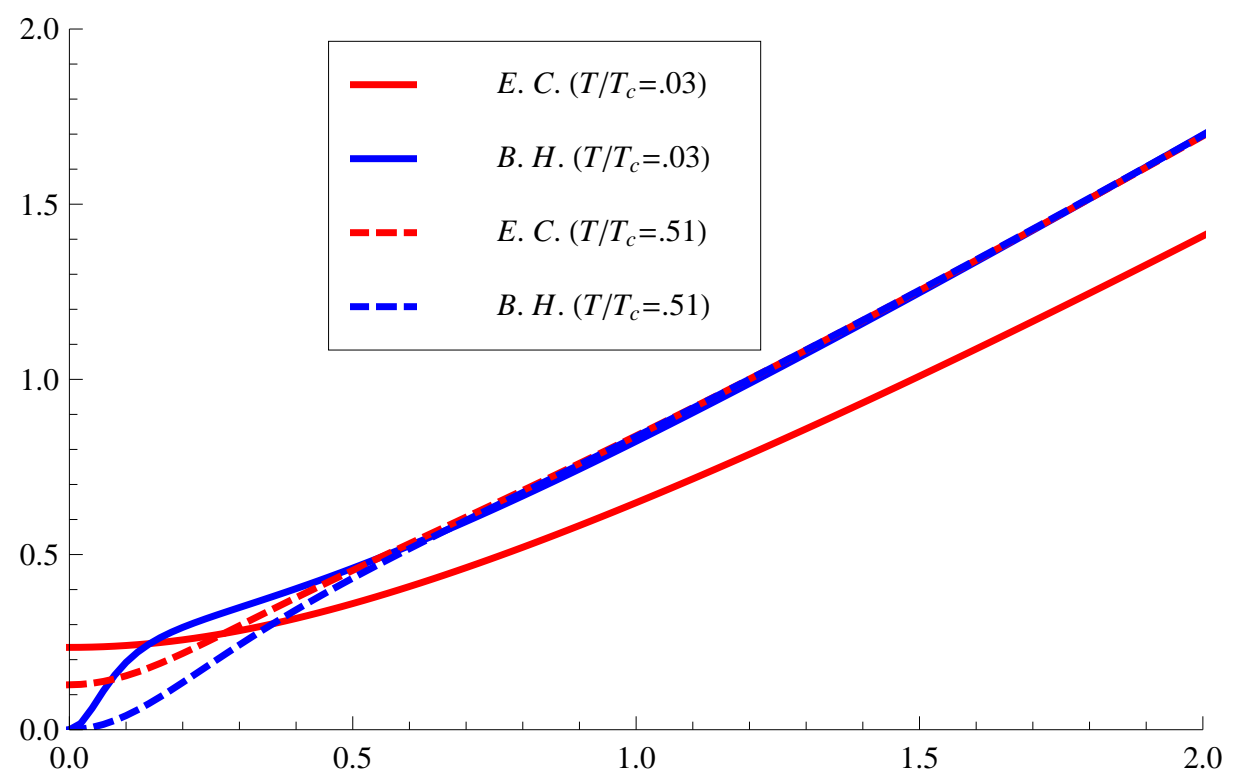

Figure 4. Classical contribution to static correlation function in the presence of a fluid with parameters $\left(\hat{\beta}=10, \hat{m}=\frac{55}{100}\right)$ and for RN black holes at moderate and low temperatures in units of $T_{c}$.

As in the AdS hard wall example we need to consider vacuum polarization effects. Indeed, neither the differential equation (4.7) nor the boundary conditions can know about a Fermi surface. Within classical fluid response, there is nowhere for nonanalyticity in the boundary momenta to enter the problem, and, accordingly, there is no evidence of a sharp Fermi surface in Figure 4. A similar result was found in [28] for the $D 4-D 8-\overline{D 8}$ system. 


\subsection{Polarized Electron Star}

We would like to include polarization effects on the shear modes' equations of motion. This represents a deviation from the idealized perfect fluid modeling. Even in flat space it is a complicated problem to go from a microscopic field theory description to a long wavelength hydrodynamical picture. In the case at hand we have the added challenge that the radial and boundary momenta enter the problem at different levels. Boundary momenta are essentially arbitrary, being fixed by boundary conditions, whereas bulk momenta are set by the scale of variation in the bulk space time. Here we will only be interested in effects which lead to non-analyticity and bulk Friedel oscillations, and will ignore other, possibly larger, effects of interactions that only contribute analytic terms to the polarization tensor.

Noting that the vacuum polarization is a short distance effect when compared to the scale of variation in the radial direction, we will introduce a poor man's modeling of the polarization,

$$
S_{P o l}=\frac{e^{2}}{2} \int d z d z^{\prime} d^{2} k \sqrt{|g(z)|\left|g\left(z^{\prime}\right)\right|} \delta A_{\mu}(z,-k) \Pi_{C G, E C}^{\mu \nu}\left(z, z^{\prime}, k\right) \delta A_{\nu}\left(z^{\prime}, k\right)
$$

where $\Pi_{C G, E C}^{\mu \nu}$ is a radially coarse grained polarization tensor. To obtain an approximate value for $\Pi_{C G, E C}^{\mu \nu}$, we start from the polarization tensor for relativistic fermions in each local Lorentz frame, $\Pi^{\mu \nu}$. Inside the fluid, we will only be interested in the behavior of the polarization tensor at long wavelengths in the radial direction. We define a coarse grained polarization tensor in flat space by simply projecting on to infinite wavelength,

$$
\begin{aligned}
\Pi_{C G, \text { flat }}^{\mu \nu}\left(k_{z}, k_{L . L .}\right) & \equiv \Pi^{\mu \nu}\left(0, k_{L . L .}\right), \\
\rightarrow \Pi_{C G, \text { flat }}^{\mu \nu}\left(z, z^{\prime}, k_{L . L .}\right) & =\delta\left(z-z^{\prime}\right) \Pi^{\mu \nu}\left(0, k_{L . L .}\right),
\end{aligned}
$$

where the spatial momentum in each local frame is denoted $k_{L . L .}$.

Locally, we then approximate the (coarse grained) curved space polarization tensor by pulling back the (coarse grained) flat space expression, using the local values of the chemical potential and Fermi momentum. This last step introduces a subtlety by redshifting the spatial momentum which is to be used in the polarization tensor. Because the fluid in each patch naturally lives in the local Lorentz frame, the momentum flowing through the polarization tensor is that of the local Lorentz frame, $k_{L . L}=z k$. Pulling all this together, we arrive at the polarization contribution to the effective action

$$
S_{P o l}=\frac{e^{2}}{2} \int d z \sqrt{|g|} \delta A_{\mu}(z,-k) \Pi^{\mu \nu}(0, z k) \delta A_{\nu}(z, k),
$$

written in terms of the flat space polarization tensor evaluated in each fluid patch. The full expressions are quite lengthy and may be found in Appendix C. 


\subsubsection{Lack of Friedel Oscillations}

As in Section 2.1, we are only interested in the additional polarization due to the finite density of fermionic matter. For brevity we will suppress all contributions not relevant for triggering Friedel oscillations. In each local patch, relativistic Friedel oscillations are caused by a mildly singular term (see Appendix C for details),

$$
\Pi^{\mu \nu}\left(0, k_{L . L .}\right) \sim N^{\mu \nu}\left(k_{L . L .}\right) \ln \left(\frac{k_{L . L .}-2 k_{f}}{k_{L . L .}+2 k_{f}}\right)+\ldots
$$

where $N^{\mu \nu}$ has smooth dependence on the momentum near $k_{f}$ and the neglected terms are also smooth at $k_{f}$. With this, the relevant term in the gauge field effective action becomes

$$
S_{P o l} \sim \frac{e^{2}}{2} \int d z \sqrt{|g|} \delta A_{\mu}(z,-k)\left[N^{\mu \nu}(z k) \ln \left(\frac{z k-2 \sqrt{\frac{(z h(z))^{2}}{f(z)}-\hat{m}^{2}}}{z k+2 \sqrt{\frac{(z h(z))^{2}}{f(z)}-\hat{m}^{2}}}\right)\right] \delta A_{\nu}(z, k) .
$$

There is an important difference between this polarization tensor and that of Section 3.3. In the hard wall example, the source of the bulk Friedel oscillations was $z$ independent. This led to a factorization of radial and boundary information such that the bulk Friedel oscillations were coherent across the radial direction. For the electron star, the bulk Fermi momentum is a local concept. Even in the deep interior of an electron star, where the local chemical potential goes to a constant, the polarization is $z$ dependent,

$$
S_{\text {Pol }} \sim \frac{e^{2}}{2} \int_{\text {large } z} d z \sqrt{|g|} \delta A_{\mu}(z,-k)\left[N^{\mu \nu}(z k) \ln \left(\frac{z k-2 \sqrt{h_{\infty}^{2}-\hat{m}^{2}}}{z k+2 \sqrt{h_{\infty}^{2}-\hat{m}^{2}}}\right)\right] \delta A_{\nu}(z, k) .
$$

We see that different bulk oscillations are triggered at each radius.

The absence of boundary Friedel oscillations in electron stars has two basic causes: there is a continuum of bulk Fermi surfaces and each surface is localized in the bulk. These facts are manifest in the smearing in two ways. First, the argument of the polarization is a function of the background profiles for the metric and gauge field. Given this, one can expect a degree of smoothing set by the sharpness of the transition from the interior Lifshitz to the asymptotic AdS behavior. The second, and more important, manifestation is the momentum redshift factor which persists all the way into the Lifshitz scaling regime. This is analogous to the smearing described in [19]. Since the fate of a continuum of bulk Fermi surfaces may be of relevance for holographic model building, it is important to understand to what extent the two sources of smearing we have identified in this work are independent. 
One might complain that the polarization effects were included in an ad hoc manner in this section. However, the approximations we have made are more or less optimal for seeing Friedel oscillations and yet there is still smearing due to the infinite number of bulk Fermi surfaces. A more thorough analysis would keep track of the radial scale over which the fermions are integrated out, almost certainly leading to even less sharp features than seen with the simple projection used here.

\section{Discussion}

In this paper, we have initiated a study of Fermi surfaces in holographic metallic states, without reference to probe fermions. Specifically, we use static currentcurrent correlation functions to characterize boundary Fermi surfaces, focusing on the question of when bulk Friedel oscillations trigger boundary Friedel oscillations. We discussed two systems of charged bulk matter, not hidden behind horizons: fermions in a hard wall truncation of AdS and an electron star geometry. For both of these systems a Luttinger count indicates that the dual theory has filled Fermi surfaces and for both systems it is necessary to consider loop effects in the current-current susceptibilities to see potential oscillations.

Fermions in the confining model geometry clearly display bulk Friedel oscillations. Because confinement leads to discrete bulk Fermi surfaces and each bulk Fermi surface is non-local in the radial direction, the bulk oscillations map into boundary Friedel oscillations. The loop parameter, " $1 / N$," sets the amplitude of the boundary Friedel oscillations, but not the wave vector at which they occur.

For the electron star, on the other hand, the bulk Fermi surfaces are local features which vary along the radial direction. We find that the local character of the bulk Fermi surface prevents bulk Friedel oscillations from combining coherently. Each radial position sources a Friedel oscillation at a different boundary momentum. This is consistent with a boundary theory comprised of a continuum of Fermi surfaces, as proposed in [19].

Extrapolating from these two examples a stronger claim would be: For holographic metallic states to exhibit a discrete set of boundary Fermi surfaces in all channels, there must be a discrete set of bulk Fermi surfaces and each bulk Fermi surfaces must be completely delocalized in the bulk. If either of these conditions fails any sharp features will be washed out.

In this paper we only analyze systems with either completely delocalized bulk Fermi surfaces or completely localized surfaces. Testing whether the lessons learned from our two examples are general is of obvious importance. An alternative system is provided by the AdS black hole with Dirac hair [18] and it would be interesting to obtain the static susceptibility for this case as well. It would also be interesting to repeat the analysis of fermions in a hard wall geometry with alternate boundary conditions. As discussed in [25], these should be dual to non-Fermi liquids and it 
would be instructive to see how this simple change causes such drastic effects in the boundary theory. Finally, it would be instructive to incorporate the ideas of [25] in a more realistic model of confinement [33] and to see the effect of Fermi surfaces on other transport quantities.

\section{Acknowledgments}

We would like to thank E. J. Brynjolfsson, J. Hertz, N. Jokela, E. Keski-Vakkuri, S. Sachdev, K. Schalm, K. P. Yogendran, and K. Zarembo for helpful discussions. This work was supported in part by the Icelandic Research Fund and by the University of Iceland Research Fund.

\section{A Perturbative Polarization Tensor}

In Section 2.1 we summarized the perturbative computation of the non-relativistic static density-density polarization,

$$
\Pi\left(x t, x^{\prime} t^{\prime}\right)=-i e^{2} \mathcal{G}\left(x t, x^{\prime} t^{\prime}\right) \mathcal{G}\left(x^{\prime} t^{\prime}, x t\right)
$$

This is textbook material (see [31] for example) and details are only presented in this Appendix to make the paper more self-contained.

As usual, it is simplest to analyze the polarization in momentum space,

$$
\Pi(q, \nu)=-i e^{2} \int \frac{d \omega d \vec{k}}{(2 \pi)^{D+1}} \mathcal{G}(k, \omega) \mathcal{G}(k+q, \omega+\nu) .
$$

Looking at the denominators of the time ordered Green's function (2.2) shows that only when $k$ and $k+q$ lie on opposite sides of $k_{f}$ will the answer be non-zero,

$$
\begin{aligned}
& \Pi(q, \nu)=e^{2} \int \frac{d \vec{k}}{(2 \pi)^{D}}\left[\frac{\theta\left(|q+k|-k_{f}\right) \theta\left(k_{f}-|k|\right)}{\nu-\left(E_{k+q}-E_{k}\right)+i \eta}-\frac{\theta\left(|k|-k_{f}\right) \theta\left(k_{f}-|q+k|\right)}{\nu-\left(E_{k+q}-E_{k}\right)-i \eta}\right] \\
&=e^{2} \int \frac{d \vec{k}}{(2 \pi)^{D}} \theta\left(|q+k|-k_{f}\right) \theta\left(k_{f}-|k|\right) \\
& \times\left(\frac{1}{\nu-\left(E_{k+q}-E_{k}\right)+i \eta}-\frac{1}{\nu+\left(E_{k+q}-E_{k}\right)-i \eta}\right) .
\end{aligned}
$$

In the second term we have changed momentum variables $k \rightarrow-k-q$ and assumed that the spectrum is invariant under sign changes (that the Fermi surface is spherical). 
The next step is to extract the imaginary part $^{8}$

$$
\begin{aligned}
\operatorname{Im} \Pi(q, \nu)=- & \pi e^{2} \int \frac{d \vec{k}}{(2 \pi)^{D}} \theta\left(|q+k|-k_{f}\right) \theta\left(k_{f}-|k|\right) \\
& \times\left[\delta\left(\nu-\left(E_{k+q}-E_{k}\right)\right)+\delta\left(\nu+\left(E_{k+q}-E_{k}\right)\right)\right] .
\end{aligned}
$$

\section{A.1 Static Limit}

If we let $\nu \rightarrow 0$ with $q$ fixed, we immediately see

$$
\operatorname{Im} \Pi(q, 0)=0
$$

There are two Fermi spheres in the problem. The step functions force you to be inside one and outside the other. On the other hand, the delta functions force the Fermi spheres to coincide and there is no solution to the constraints.

From the static real part we have

$$
\begin{aligned}
\operatorname{Re} \Pi(q, 0) & =-e^{2} \mathcal{P} \int \frac{d \vec{k}}{(2 \pi)^{D}}\left[1-\theta\left(k_{f}-|k+q|\right)\right] \theta\left(k_{f}-|k|\right) \frac{2}{\left(E_{k+q}-E_{k}\right)} \\
& =-e^{2} \mathcal{P} \int \frac{d \vec{k}}{(2 \pi)^{D}} \theta\left(k_{f}-|k|\right) \frac{2}{\left(E_{k+q}-E_{k}\right)} .
\end{aligned}
$$

Here we have used antisymmetry under $k \leftrightarrow k+q$. In $2+1$ dimensions we have

$$
\operatorname{Re} \Pi(q, 0)=-\frac{e^{2}}{2 \pi^{2}} \mathcal{P} \int d k d \theta \theta\left(k_{f}-k\right) \frac{k}{E_{k+q}-E_{k}} .
$$

For spherical Fermi surfaces the integrand is singular when $q=2 k_{f}$. In general, the polarization tensor has non-analytic behavior in the momentum space and oscillatory behavior in position space.

For example, for non-relativistic fermions we have $E_{k+q}-E_{k}=q^{2} / 2 m+k q \cos (\theta) / m$. The polarization becomes,

$$
\begin{aligned}
\operatorname{Re} \Pi(q, 0) & =-\frac{m e^{2}}{2 \pi^{2} q} \mathcal{P} \int d k d \theta \theta\left(k_{f}-k\right) \frac{k}{q / 2+k \cos (\theta)} \\
& =-\frac{m e^{2}}{2 \pi}\left(1-\sqrt{1-\left(\frac{2 k_{f}}{q}\right)^{2}} \theta\left(q-2 k_{f}\right)\right) .
\end{aligned}
$$

\footnotetext{
${ }^{8}$ Recall that for real $\omega$
}

$$
\frac{1}{\omega-\omega^{\prime} \pm i \eta}=\mathcal{P} \frac{1}{\omega-\omega^{\prime}} \mp \pi i \delta\left(\omega-\omega^{\prime}\right)
$$




\section{B Holographic Relative Polarization Tensor}

In Section 2.1 we introduced the relative polarization tensor,

$$
\begin{array}{r}
\Pi_{r e l}^{\mu \nu}\left(z, z^{\prime}, \vec{p}\right)=-\int \frac{d \omega d^{2} k}{(2 \pi)^{3}} \operatorname{Tr}\left(M^{\mu} G\left(\omega, k, z, z^{\prime}\right) M^{\nu} G\left(\omega, k+p, z^{\prime}, z\right)\right) \\
-\operatorname{Tr}\left(M^{\mu} G^{0}\left(\omega, k, z, z^{\prime}\right) M^{\nu} G^{0}\left(\omega, k+p, z^{\prime}, z\right)\right) .
\end{array}
$$

Using Eq. (3.4) we can perform the frequency integral:

$$
\begin{aligned}
& \Pi_{r e l}^{\mu \nu}\left(z, z^{\prime}, \vec{p}\right)= \\
& \sum_{\ell \ell^{\prime}}{ }^{\prime} \int \frac{d^{2} k}{(2 \pi)^{2}}\left[\frac{\theta\left(E_{\ell^{\prime}}(k+p)\right) \theta\left(-E_{\ell}(k)\right)}{E_{\ell^{\prime}}(k+p)-E_{\ell}(k)+i \eta \mathrm{s}\left(E_{\ell}(k)\right)}-\frac{\theta\left(-E_{\ell^{\prime}}(k+p)\right) \theta\left(E_{\ell}(k)\right)}{E_{\ell^{\prime}}(k+p)-E_{\ell}(k)-i \eta \mathrm{s}\left(E_{\ell}(k)\right)}\right] \\
& \quad \times \operatorname{Tr} M^{\mu} \chi_{\ell, k}(z) \chi_{\ell, k}^{\dagger}\left(z^{\prime}\right) M^{\nu} \chi_{\ell^{\prime}, k+p}\left(z^{\prime}\right) \chi_{\ell^{\prime}, k+p}^{\dagger}(z) \\
& -\int \frac{d^{2} k}{(2 \pi)^{2}}\left[\frac{\theta\left(\ell^{\prime}\right) \theta(-\ell)}{E_{\ell^{\prime}, k+p}^{0}-E_{\ell, k}^{0}-i \eta}-\frac{\theta\left(-\ell^{\prime}\right) \theta(\ell)}{E_{\ell^{\prime}, k+p}^{0}-E_{\ell, k}^{0}-i \eta}\right] \\
& \quad \times \operatorname{Tr} M^{\mu} \chi_{\ell, k}^{0}(z) \chi_{\ell, k}^{0 \dagger}\left(z^{\prime}\right) M^{\nu} \chi_{\ell^{\prime}, k+p}^{0}\left(z^{\prime}\right) \chi_{\ell^{\prime}, k+p}^{0 \dagger}(z) .
\end{aligned}
$$

In this we have denoted the contributions with zero chemical potential with ${ }^{0}$ and the primed summation indicates that the mode sums have no $\ell, \ell^{\prime}=0$ terms. Also note that we have introduced the notation $s(x) \equiv \operatorname{sign}(x)$.

Though expression in Eq. (B.2) is quite complicated, there are two important features to note. Firstly, it is finite in the bulk UV. The large momentum and radial mode contributions do not know about the low energy finite density physics. Secondly, spherical bulk Fermi surfaces imply singular behavior due to the integrand's denominator at $p=2 k_{f}$ and $\ell=\ell^{\prime}$. The only terms where $\ell=\ell^{\prime}$ are when $k$ and $k+p$ lie on opposite sides of a Fermi surface. Quite generally, these singularities in the integrand will source Friedel oscillations in position space.

\section{B.1 High Confinement Scale and Non-Relativistic Limit}

We will now consider a simplifying situation where there is a single Fermi surface occurring at $k=k_{f}$ and $\ell=1$. In this case we may split the summand in Eq. (B.2) into two classes, $\ell=\ell^{\prime}=1$ and all other combinations,

$$
\begin{aligned}
& \Pi_{r e l}^{\mu \nu}\left(z, z^{\prime}, \vec{p}\right)= \\
& \int \frac{d^{2} k}{(2 \pi)^{2}}\left(\frac{\theta\left(|k+p|-k_{f}\right) \theta\left(k_{f}-|k|\right)}{E_{1}(k+p)-E_{1}(k)+i \eta \mathrm{s}\left(E_{1}(k)\right)}-\frac{\theta\left(k_{f}-|k+p|\right) \theta\left(|k|-k_{f}\right)}{E_{1}(k+p)-E_{1}(k)-i \eta \mathrm{s}\left(E_{1}(k)\right)}\right) \\
& \quad \times \operatorname{Tr} M^{\mu} \chi_{1, k}(z) \chi_{1, k}^{\dagger}\left(z^{\prime}\right) M^{\nu} \chi_{1, k+p}\left(z^{\prime}\right) \chi_{1, k+p}^{\dagger}(z)+\Pi_{\text {analytic }}^{\mu \nu} .
\end{aligned}
$$

If $\ell=\ell^{\prime}=1$ the integrand has a mild singularity due to the Fermi surface and there is no contribution from the vacuum terms. The rest of the mode number sums are analytic in the external momenta and do not involve the Fermi surface. 
It is difficult to deal with the analytic contributions without explicit expressions for the energy eigenvalues and wave functions. However, we do note that their dominant contributions come from small momenta where the energy values scale with $E_{\ell}(0) \sim \frac{1}{z_{m}}$. Consequently, the analytic contributions to the relative vacuum polarization scale as $z_{m}$. If we model a high confinement scale, we can safely ignore all but the $\ell=\ell^{\prime}=1$ contributions,

$$
\begin{aligned}
& \Pi_{r e l}^{\mu \nu}\left(z, z^{\prime}, \vec{p}\right)= \\
& \int \frac{d^{2} k}{(2 \pi)^{2}}\left(\frac{\theta\left(|k+p|-k_{f}\right) \theta\left(k_{f}-|k|\right)}{E_{1}(k+p)-E_{1}(k)+i \eta \mathrm{s}\left(E_{1}(k)\right)}-\frac{\theta\left(k_{f}-|k+p|\right) \theta\left(|k|-k_{f}\right)}{E_{1}(k+p)-E_{1}(k)-i \eta \mathrm{s}\left(E_{1}(k)\right)}\right) \\
& \quad \times \operatorname{Tr} M^{\mu} \chi_{1, k}(z) \chi_{1, k}^{\dagger}\left(z^{\prime}\right) M^{\nu} \chi_{1, k+p}\left(z^{\prime}\right) \chi_{1, k+p}^{\dagger}(z)+\mathcal{O}\left(z_{m}\right) .
\end{aligned}
$$

While this is a great simplification, it is not quite enough for us to be able to find an analytic expression. In order to go further we will take a non-relativistic limit and scale the chemical potential towards the bulk mass. In this way we are left with a small $k_{f}$ and numerically observe that, for small momenta, the energy spectrum is well approximated by,

$$
E_{1}(k) \sim a_{1}+b_{1} k^{2} .
$$

Using this functional form the holographic calculation closely parallels the $2+1$ non-relativistic example in Section A.1 with $2 b_{1}$ playing the role of an inverse mass,

$$
\begin{aligned}
& \Pi_{r e l}^{\mu \nu}\left(z, z^{\prime}, \vec{p}\right)= \\
& \frac{1}{p b_{1}} \int \frac{d^{2} k}{(2 \pi)^{2}}\left[\frac{\theta\left(E_{1}(k+p)\right) \theta\left(-E_{1}(k)\right)}{p+2 \cos (\theta) k+i \eta \mathrm{s}\left(E_{1}(k)\right)}\right. \\
& \quad \times \operatorname{Tr}\left(M^{\mu} \chi_{1, k}(z) \chi_{1, k}^{\dagger}\left(z^{\prime}\right) M^{\nu} \chi_{1, k+p}\left(z^{\prime}\right) \chi_{1, k+p}^{\dagger}(z)\right) \\
& \left.-\frac{\theta\left(E_{1}(k-p)\right) \theta\left(-E_{1}(k)\right)}{p-2 \cos (\theta) k-i \eta \mathrm{s}\left(E_{1}(k)\right)} \operatorname{Tr}\left(M^{\mu} \chi_{1, k-p}(z) \chi_{1, k-p}^{\dagger}\left(z^{\prime}\right) M^{\nu} \chi_{1, k}\left(z^{\prime}\right) \chi_{1, k}^{\dagger}(z)\right)\right] .
\end{aligned}
$$

As in the non-relativistic case, the static polarization is purely real. We have

$$
\begin{aligned}
\Pi_{r e l}^{\mu \nu}\left(z, z^{\prime}, \vec{p}\right)= & \frac{1}{4 b_{1} \pi^{2} p} \mathcal{P} \int d k d \theta \theta\left(k_{f}-k\right) \frac{k}{p / 2+k \cos (\theta)} \\
& \times \operatorname{Tr}\left(M^{\mu} \chi_{1, k}(z) \chi_{1, k}^{\dagger}\left(z^{\prime}\right) M^{\nu} \chi_{1, k+p}\left(z^{\prime}\right) \chi_{1, k+p}^{\dagger}(z)\right) .
\end{aligned}
$$

Numerically, it is expensive to work with Eq. (B.7) without further approximation. We can use the fact that $k_{f}$ is small which means that the magnitude of the loop momentum is never large and that the wave functions are slowly varying functions of the momenta. Therefore we may expand the wave function factors for small loop momenta, $k$,

$$
\begin{aligned}
& \operatorname{Tr}\left(M^{0} \chi_{1, k}(z) \chi_{1, k}^{\dagger}\left(z^{\prime}\right) M^{0} \chi_{1, k+p}\left(z^{\prime}\right) \chi_{1, k+p}^{\dagger}(z)\right) \\
& \sim \operatorname{Tr}\left(M^{0} \chi_{1,0}(z) \chi_{1,0}^{\dagger}\left(z^{\prime}\right) M^{0} \chi_{1, p}\left(z^{\prime}\right) \chi_{1, p}^{\dagger}(z)\right) .
\end{aligned}
$$


This approximation loses some of the angular information in the wavefunctions, but is sufficient to display the essential features. In this way we find,

$$
\begin{aligned}
\Pi_{r e l}^{\mu \nu}\left(z, z^{\prime}, \vec{p}\right)= & \frac{1}{4 b_{1} \pi^{2} p} \operatorname{Tr}\left(M^{\mu} \chi_{1,0}(z) \chi_{1,0}^{\dagger}\left(z^{\prime}\right) M^{\nu} \chi_{1, p}\left(z^{\prime}\right) \chi_{1, p}^{\dagger}(z)\right) \\
& \times \mathcal{P} \int d k d \theta \theta\left(k_{f}-k\right) \frac{k}{p / 2+k \cos (\theta)}
\end{aligned}
$$

Introducing the effective expansion parameter, $\lambda \equiv \frac{1}{4 \pi b_{1}}$,

$$
\begin{aligned}
\Pi_{r e l}^{\mu \nu}\left(z, z^{\prime}, \vec{p}\right)= & -\lambda \operatorname{Tr}\left(M^{\mu} \chi_{1,0}(z) \chi_{1,0}^{\dagger}\left(z^{\prime}\right) M^{\nu} \chi_{1, p}\left(z^{\prime}\right) \chi_{1, p}^{\dagger}(z)\right) \\
& \times\left(1-\sqrt{1-\left(\frac{2 k_{f}}{p}\right)^{2}} \theta\left(p-2 k_{f}\right)\right)
\end{aligned}
$$

This is the form of the polarization used in Section 3.4.1.

\section{Flat Space QED Polarization Tensor}

We can understand the emergence of Friedel oscillations in relativistic Fermi liquid theory by studying how weakly coupled relativistic fermions respond to changes in an external gauge field. When working at finite chemical potential, the Lorentz invariance is broken and the polarization tensor may only be decomposed using the residual spatial rotation symmetry. ${ }^{9}$ If we introduce projections onto spatial directions, $P_{L}$, and the transverse direction, $P_{T}$, we may write

$$
\Pi^{\mu \nu}=G P_{T}^{\mu \nu}+F P_{L}^{\mu \nu}
$$

Expressing the projectors in terms of the spatial momenta, we have

$$
\begin{aligned}
P_{T}^{i j} & =\delta^{i j}-\frac{\vec{k}^{i} \vec{k}^{j}}{\vec{k}^{2}} \quad(\text { only spatial directions } \neq 0) \\
P_{L}^{\mu \nu} & =k^{\mu} k^{\nu} / k^{2}-g^{\mu \nu}-P_{L}^{\mu \nu}
\end{aligned}
$$

As is mentioned in Section 3.3 in finite density computations, it is useful to further split the polarization tensor into a "vacuum" part and the relative, finite density, contribution,

$$
\begin{aligned}
\Pi^{\mu \nu} & =\Pi_{v a c}^{\mu \nu}+\Pi_{r e l}^{\mu \nu}, \\
\Pi_{v a c}^{\mu \nu} & =\left.\Pi^{\mu \nu}\right|_{\mu, T \rightarrow 0} .
\end{aligned}
$$

\footnotetext{
${ }^{9}$ For a general reference to relativistic field theories at finite temperature/density see [30].
} 
The vacuum contribution is a standard textbook computation [30]. Focusing on $3+1$ dimensions, $F(0, \vec{k})$, may be found in [30, 37],

$$
\begin{aligned}
\left(\frac{24 \pi^{2}}{e^{2}}\right) F_{r e l}(0, k)= & 16 \mu k_{f}+4 k^{2} \ln \left(\frac{m}{\mu+k_{f}}\right) \\
& +\left(\frac{\mu}{k}\right)\left(3 k^{2}-4 \mu^{2}\right) \ln \left(\frac{k-2 k_{f}}{k+2 k_{f}}\right)^{2} \\
& +\left(\frac{2 m^{2}-k^{2}}{k}\right) \sqrt{k^{2}+4 m^{2}} \ln \left(\frac{\sqrt{k^{2}+4 m^{2}} k_{f}-k \mu}{\sqrt{k^{2}+4 m^{2}} k_{f}-k \mu}\right)^{2} .
\end{aligned}
$$

A similar straightforward calculation yields

$$
\begin{aligned}
\left(\frac{24 \pi^{2}}{e^{2}}\right) G_{r e l}(0, k)= & 4 \mu k_{f}-8 k^{2} \ln \left(\frac{m}{\mu+k_{f}}\right) \\
& +\mu\left(\frac{4 \mu^{2}-12 m^{2}-9 k^{2}}{2 k}\right) \ln \left(\frac{k-2 k_{f}}{k+2 k_{f}}\right)^{2} \\
& -\frac{\left(4 m^{2}+k^{2}\right)^{3 / 2}}{k} \ln \left(\frac{\sqrt{k^{2}+4 m^{2}} k_{f}-\mu k}{\sqrt{k^{2}+4 m^{2}} k_{f}+\mu k}\right)^{2} .
\end{aligned}
$$

The vacuum contributions contain the usual ultraviolet divergences for QED, and contain no information about the Fermi surface which is set by $\mu$. For us, the most important feature is the logarithmic term with a singularity at $k=2 k_{f}$ in both of these functions. Upon Fourier transformation this gives rise to spatial variations with wave number $2 k_{f}$. When using this in the holographic calculation one should note that the appropriate values of $k$ and $k_{f}$ are functions of the radial coordinate through redshift factors.

\section{References}

[1] S. A. Hartnoll, "Horizons, holography and condensed matter," arXiv:1106.4324 [hep-th] .

[2] S. Sachdev, "What can gauge-gravity duality teach us about condensed matter physics?," 1108.1197 [cond-mat.str-el] .

[3] N. Iqbal, H. Liu, M. Mezei, "Lectures on holographic non-Fermi liquids and quantum phase transitions," arXiv:1110.3814 [hep-th].

[4] J. Polchinski, "Effective field theory and the Fermi surface," arXiv:hep-th/9210046.

[5] R. Shankar, "Renormalization group approach to interacting fermions," Rev. Mod. Phys. 66, 129 (1994), arXiv: cond-mat/9307009

[6] S.-S. Lee, "Low energy effective theory of Fermi surface coupled with a U(1) gauge field in 2+1 dimensions," Phys. Rev. B 80, 165102 (2009), arXiv:0905.4532 [cond-mat.str-el] . 
[7] M.A. Metlitski and S. Sachdev, "Quantum phase transitions of metals in two spatial dimensions: I Ising-nematic order," Phys. Rev. B 82, 075127 (2010), arXiv:1001.1153 [cond-mat.str-el].

[8] M.A. Metlitski and S. Sachdev, "Quantum phase transitions of metals in two spatial dimensions: II Spin density wave order," Phys. Rev. B 82, 075128 (2010), arXiv:1005.1288 [cond-mat.str-el].

[9] S. -S. Lee, "A Non-Fermi Liquid from a Charged Black Hole: A Critical Fermi Ball," Phys. Rev. D79, 086006 (2009), arXiv:0809.3402 [hep-th].

[10] H. Liu, J. McGreevy, D. Vegh, "Non-Fermi liquids from holography," Phys. Rev. D83, 065029 (2011), arXiv:0903.2477 [hep-th] .

[11] T. Faulkner, H. Liu, J. McGreevy, D. Vegh, "Emergent quantum criticality, Fermi surfaces, and AdS(2)," Phys. Rev. D83, 125002 (2011), arXiv:0907. 2694 [hep-th] .

[12] M. Cubrovic, J. Zaanen, K. Schalm, "String Theory, Quantum Phase Transitions and the Emergent Fermi-Liquid," Science 325, 439-444 (2009), arXiv:0904.1993 [hep-th] .

[13] F. Denef, S. A. Hartnoll and S. Sachdev, "Quantum oscillations and black hole ringing," Phys. Rev. D 80 (2009) 126016, arXiv:0908.1788 [hep-th] .

[14] S. A. Hartnoll, J. Polchinski, E. Silverstein, D. Tong, "Towards strange metallic holography," JHEP 1004 (2010) 120, arXiv:0912.1061 [hep-th].

[15] T. Faulkner, J. Polchinski, "Semi-Holographic Fermi Liquids," JHEP 1106, 012 (2011), arXiv:1001.5049 [hep-th].

[16] S. Sachdev, "Holographic metals and the fractionalized Fermi liquid," Phys. Rev. Lett. 105 (2010) 151602, arXiv:1006.3794 [hep-th] .

[17] S. A. Hartnoll, A. Tavanfar, "Electron stars for holographic metallic criticality," Phys. Rev. D83 (2011) 046003. arXiv:1008.2828 [hep-th].

[18] M. Cubrovic, J. Zaanen and K. Schalm, "Constructing the AdS dual of a Fermi liquid: AdS Black holes with Dirac hair," arXiv:1012.5681 [hep-th] .

[19] S. A. Hartnoll, D. M. Hofman, A. Tavanfar, "Holographically smeared Fermi surface: Quantum oscillations and Luttinger count in electron stars," Europhys. Lett. 95 (2011) 31002, arXiv:1011.2502 [hep-th] .

[20] N. Iizuka, N. Kundu, P. Narayan and S. P. Trivedi, "Holographic Fermi and Non-Fermi Liquids with Transitions in Dilaton Gravity," arXiv:1105.1162 [hep-th] .

[21] S. A. Hartnoll, D. M. Hofman, D. Vegh, "Stellar spectroscopy: Fermions and holographic Lifshitz criticality," arXiv:1105.3197 [hep-th] .

[22] X. Arsiwalla, J. de Boer, K. Papadodimas, E. Verlinde, "Degenerate Stars and Gravitational Collapse in AdS/CFT," JHEP 1101 (2011) 144, arXiv:1010.5784 
[hep-th] .

[23] M. Cubrovic, Y. Liu, K. Schalm, Y. -W. Sun, J. Zaanen, "Spectral probes of the holographic Fermi groundstate: dialing between the electron star and AdS Dirac hair," arXiv:1106.1798 [hep-th] .

[24] M. Edalati, K. W. Lo, P. W. Phillips, "Neutral Order Parameters in Metallic Criticality in d=2+1 from a Hairy Electron Star," arXiv:1106.3139 [hep-th] .

[25] S. Sachdev, "A model of a Fermi liquid using gauge-gravity duality," Phys. Rev. D84 (2011) 066009, arXiv:1107.5321 [hep-th].

[26] V. G. M. Puletti, S. Nowling, L. Thorlacius, T. Zingg, "Holographic metals at finite temperature," JHEP 1101 (2011) 117. arXiv:1011.6261 [hep-th].

[27] S. A. Hartnoll, P. Petrov, "Electron star birth: A continuous phase transition at nonzero density," Phys. Rev. Lett. 106 (2011) 121601. arXiv:1011.6469 [hep-th] .

[28] M. Kulaxizi, A. Parnachev, "Holographic Responses of Fermion Matter," Nucl. Phys. B815 (2009) 125-141, arXiv:0811.2262 [hep-th] .

[29] S. Powell, S. Sachdev, H. P. Buchler, "Depletion of the Bose-Einstein condensate in Bose-Fermi mixtures," Phys. Rev. B72 (2005) 024534, arXiv:cond-mat/0502299

[30] J. I. Kapusta, C. Gale, "Finite-temperature field theory: Principles and applications," Cambridge, UK: Univ. Pr. (2006) 428 p.

[31] A. L. Fetter, J. D. Walecka, "Quantum theory of many-particle systems," Dover Pubns. (2003) 601p.

[32] J. Erlich, E. Katz, D. T. Son and M. A. Stephanov, "QCD and a holographic model of hadrons," Phys. Rev. Lett. 95 (2005) 261602 arXiv:hep-ph/0501128.

[33] E. Witten, "Anti-de Sitter space, thermal phase transition, and confinement in gauge theories," Adv. Theor. Math. Phys. 2 (1998) 505-532, arXiv:hep-th/9803131.

[34] L. Huijse, S. Sachdev, "Fermi surfaces and gauge-gravity duality," Phys. Rev. D84 (2011) 026001, arXiv:1104.5022 [hep-th].

[35] M. Edalati, J. I. Jottar, R. G. Leigh, "Shear Modes, Criticality and Extremal Black Holes," JHEP 1004 (2010) 075. arXiv:1001.0779 [hep-th] .

[36] M. Edalati, J. I. Jottar, R. G. Leigh, "Holography and the sound of criticality," JHEP 1010 (2010) 058. arXiv:1005.4075 [hep-th] .

[37] J. I. Kapusta, T. Toimela, "Friedel Oscillations In Relativistic QED And QCD," Phys. Rev. D37 (1988) 3731. 